OU-HET 371

MCTP-00-15

hep-th/0012239

\title{
The Galilean Nature of V-duality for Noncommutative Open String and Yang-Mills Theories
}

\author{
Rong-Gen Cai', J. X. Lu田, and Yong-Shi Wu角 \\ * Department of Physics, Osaka University, Toyonaka, Osaka 560-0043, Japan \\ ${ }^{\dagger}$ Michigan Center for Theoretical Physics, Randall Physics Laboratory \\ University of Michigan, Ann Arbor MI 48109-1120, USA \\ ¥ Department of Physics, University of Utah, Salt Lake City, Utah 84112, USA
}

\begin{abstract}
A V-duality conjecture for noncommutative open string theories (NCOS) that result from decoupling D-branes in Lorentz-boost related backgrounds was put forward recently in hepth/0006013. The aim of this paper is to test the Galilean nature of this conjecture in the gravity dual setup. We start with an (F, D3) bound state Lorentz-boosted along one D3-brane direction perpendicular to the F-string, and show that insisting a decoupled NCOS allows only infinitesimal Lorentz boosts. In this way, it is shown that the $\mathrm{V}$-duality relates a family of NCOS by Galileo boosts. Starting with a Lorentz-boosted (D1,D3) bound state, we show that a similar V-duality works for noncommutative Yang-Mills (NCYM) theories as well. In addition, we deduce by a holography argument that the running string tension, as a function of the energy scale, for NCOS (or NCYM) remains unchanged under V-duality.
\end{abstract}

\footnotetext{
*cai@het.phys.sci.osaka-u.ac.jp

$\dagger j x l u @ u m i c h . e d u$

${ }^{\ddagger}$ wu@physics.utah.edu
} 


\section{INTRODUCTION}

There is a surge of interest recently in seeking dynamical theories without gravity in string/M theory. The motivation is two-fold. On one hand, we try to understand better the Standard Model physics using the knowledge of string/M theory. One good example is the AdS/CFT correspondence and its variations. On the other hand, we intend to collect more theoretical data for the eventual formulation of M-theory since these decoupled theories are part of the big M-theory and we have a better hand on them due to the absence of gravity.

Among these decoupled theories, for the purpose of this paper, we will focus on the so-called noncommutative Yang-Mills theory (NCYM) Đ and noncommutative open string theory (NCOS) 2. In the simplest context, a $(1+\mathrm{p})$-dimensional noncommutative YangMills with space-space noncommutativity arises in the decoupling limit as the decoupled theory of Dp branes in a constant magnetic background (or constant B-field with only spatial components). However, a decoupled noncommutative field theory does not exist for Dp branes in a constant electric background. Instead, one can have a decoupled open string theory living on the Dp brane worldvolume with space-time noncommutativity in the critical electric field limit. This is consistent with the fact that a unitary field theory cannot exist with space-time noncommutativity [20,21]. The basic picture here is that in the decoupling limit the near critical electric force stretches the open string ending on the Dp brane, and balances the usual string tension to end up with almost tensionless string confined on the branes.

It is well-known that we have various dualities such as $S-$ and $T$-, or in general $U$-dualities in the big M-theory. Up to this point, these dualities are global discrete transformations which relate different but physically equivalent vacua in M-theory. These dualities are believed to be inherited in the so-called little m-theory without gravity which is obtained as a decoupled theory of M-theory. NCOS and NCYM are among this little m-theory. Because of the absence of gravity (or in general the closed strings) in these

\footnotetext{
${ }^{1}$ An incomplete list of references is [1] 8 .

${ }^{2}$ An incomplete list of references for NCOS is 9 19.

${ }^{3}$ We can keep the tension for the NCOS fixed by scaling the original tension to infinity.
} 
decoupled theories, one may wonder whether there exist some new global transformationsf also called dualities, which connect physically equivalent theories. It has been suggested in [14 that there exists a new spacetime duality relating different but physically equivalent NCOS which result from decoupling D-branes in Lorentz-boost related backgrounds. This can be understood as follows: The open string metric and space-time (and/or space-space) noncommutativity are both dictated by constant electric (and/or magnetic) background on the D-brane worldvolume. Some background configurations are related by the worldvolume Lorentz boosts. The corresponding decoupled worldvolume noncommutative theories are conjectured to be physically equivalent to each other, since the Lorentz boosts are expected to be a symmetry of the parent perturbative open string theory in the decoupling limits. In this way, some decoupled theories with different noncommutativities and/or (open-string) metrics can be mapped to each other. For example, by first applying a Lorentz boost on a purely electric background and then imposing the corresponding decoupling limit, one can obtain a $(1+\mathrm{p})$-dimensional NCOS with both space-time and space-space noncommutativities as a decoupled theory of Dp branes with constant electric and magnetic backgrounds. This NCOS is related to the original NCOS with only space-time noncommutativity [14 by the new spacetime duality. This conjectured duality was named V-duality in ref. [14], because it originates from boosts, characterized by a relative velocity, and alphabetically it follows the existing $S$-, $T$ - and $U$-dualities.

Naturally raised is the following question: What is the action of the V-duality on metric and noncommutativities? Namely, what is the transformation that connects the decoupled theories arising from decoupling Dp branes in backgrounds related by a Lorentz boost $\mathrm{T}$ ? It is not necessarily a Lorentz boost, because the decoupling limit involves intriguing scalings of the background and the (closed-string) metric. For example, consider a NCOS theory resulting from decoupling D-branes in a background which is related to a purely electric

\footnotetext{
${ }^{4}$ We exclude those transformations which belong to the symmetry group of the underlying theory

${ }^{5}$ The nature of theory (either NCOS or NCYM) cannot be changed since the Lorentz boost leaves $\mathbf{E}^{2}-\mathbf{B}^{2}$ invariant.

${ }^{6}$ We consider only those Lorentz boosts which do not leave the background fields invariant.
} 
background by a Lorentz-boost. In ref. [14] an analysis using Seiberg-Witten relations [5] indicated that the result is a Galilean boost for the Lorentz boost in a direction orthogonal to that of the electric background. This is a particular case with orthogonal electric and magnetic backgrounds.

In summary, the V-duality can be stated clearly in two parts: a) the NCOS's (or NCYM's) resulting from decoupling D-branes in Lorentz-related backgrounds are related to each other by Galileo boosts; [ and b) such related NCOS or NCYM theories are physically equivalent to each other. In this paper, we will test the V-duality from the gravity dual description of NCOS, and will find that indeed the V-duality holds exactly as suggested in 14. Further, we will extend this analysis of V-duality to NCYM theories that result from decouplings of $\mathrm{Dp}$ branes in backgrounds Lorentz-boost related to a purely magnetic background and show that the V-duality holds for NCYM as well. For concreteness, we will focus on the case of $p=3$ from now on, but the conclusion drawn is general.

In this paper, we limit ourselves to the particular cases in which the backgrounds are Lorentz-boost related to a purely electric or a purely magnetic background. However, our investigation indicates that the V-duality holds in general, for example, for NCOS and NCYM resulting from their respective decouplings of D3 branes with backgrounds Lorentz-boost related to a given parallel electric and magnetic background as discussed in 14, 15, 19. We plan to report the general discussion of V-duality and its structure, significance and relations to other theories such as Matrix string theory in a separate paper 22.

This paper is organized as follows. In section 2, we first present a gravity configuration of (F, D3) bound state boosted along a brane direction orthogonal to the F-strings. This system provides the gravity dual description of NCOS resulting from the decoupling of D3 branes in an orthogonal electric and magnetic background (time-like). We show that the gravity description is related to that of NCOS resulting from a purely electric background by a Galilean transformation. In other words, we show that the V-duality proposed in [14] holds also true in the gravity description. In section 3, we present a gravity configuration of (D1, D3) bound state boosted along a brane direction orthogonal to the D-strings. This

\footnotetext{
${ }^{7}$ Recall that the noncommutative geometry for either NCOS or NCYM is described by the (open string) metric and the (anti-symmetric) coordinate noncommutative matrix.
} 
system provides a gravity dual description of NCYM resulting from the decoupling of D3 branes in an orthogonal electric and magnetic background (space-like). We show that similar V-duality holds here almost trivially. In section 4, we give a detailed explanation of holographic correspondence proposed in 28, 14] for NCYM and NCOS. Then we give a holographic derivation of the NSNS fields in the gravity dual description of NCOS discussed in section 2. We conclude this paper in section 5 where some issues on the validity of V-duality are also addressed.

\section{THE GALILEAN NATURE OF NCOS: V-DUALITY}

We test the V-duality for NCOS using its gravity description in this section. The relevant gravity configuration can be obtained by a Lorentz boost of the (F, D3) bound state [23,24 along a D3 brane direction orthogonal to the F-strings in the bound state. The explicit form is

$$
\begin{aligned}
& d s^{2}=H^{\prime-1 / 2}\left[\frac{H^{\prime}}{H}\left(-\left(d x^{0}\right)^{2}+\left(d x^{1}\right)^{2}+\left(d x^{2}\right)^{2}\right)\right. \\
& \left.\quad+\frac{4 \pi n g_{s} \alpha^{\prime 2}}{r^{4} H} \sin \alpha \tan \alpha\left(\cosh \gamma d x^{2}-\sinh \gamma d x^{0}\right)^{2}+\left(d x^{3}\right)^{2}\right]+H^{\prime 1 / 2}\left[d r^{2}+r^{2} d \Omega_{5}^{2}\right] \\
& e^{\phi}=g_{s} \sqrt{H^{\prime} / H}, \quad F_{5}=16 \pi n \alpha^{\prime 2}\left(* \epsilon_{5}+\epsilon_{5}\right), \\
& 2 \pi \alpha^{\prime} B=\sin \alpha H^{-1}\left(\cosh \gamma d x^{0} \wedge d x^{1}+\sinh \gamma d x^{1} \wedge d x^{2}\right) \\
& A_{2}=g_{s}^{-1} \tan \alpha H^{\prime-1}\left(-\sinh \gamma d x^{0} \wedge d x^{3}+\cosh \gamma d x^{2} \wedge d x^{3}\right)
\end{aligned}
$$

where we have the metric in string frame, $\epsilon_{5}$ denotes the volume form of unit 5 -sphere, * denotes the Hodge dual in 10 dimensions, and the harmonic functions are

$$
H=1+\frac{4 \pi n g_{s} \alpha^{\prime 2}}{r^{4}} \frac{1}{\cos \alpha}, \quad H^{\prime}=1+\frac{4 \pi n g_{s} \alpha^{\prime 2}}{r^{4}} \cos \alpha .
$$

In the above, the integer $n$ is the number of D3 branes in the bound state and $g_{s}$ is the asymptotic string coupling. The boost is along the $x^{2}$ direction with boost parameter $\gamma$. When $\gamma=0$, we recover the (F, D3) bound state. We can read the electric and magnetic background fields from the asymptotic B-field (i.e., $r \rightarrow \infty$ ) from the above as $E_{1}=B_{01}^{\infty}=\sin \alpha \cosh \gamma, B_{3}=B_{12}^{\infty}=\sin \alpha \sinh \gamma$. For $\gamma=0$, we have only electric field. We have here $\mathbf{E}^{2}-\mathbf{B}^{2}=\sin ^{2} \alpha \geq 0$ and $\mathbf{E} \cdot \mathbf{B}=0$ which are invariant under a Lorentz transformation as is evident. For NCOS, we need $\sin \alpha \neq 0$, therefore $\mathbf{E}^{2}-\mathbf{B}^{2}>0$, i.e., 
time-like as it should be. The gravity dual description can be obtained from the above with the following decoupling limit:

$$
\begin{aligned}
& \alpha^{\prime}=\alpha_{\text {eff }}^{\prime} \epsilon, \quad \cos \alpha=\epsilon, \quad \gamma=\tilde{v} \epsilon, \quad g_{s}=\frac{G_{o}^{2}}{\epsilon} \\
& r=\alpha_{\text {eff }}^{\prime} \epsilon^{1 / 2} u, \quad x^{0,1}=\frac{\tilde{x}^{0,1}}{\sqrt{\epsilon}}, x^{2,3}=\sqrt{\epsilon} \tilde{x}^{2,3}
\end{aligned}
$$

where $\epsilon \rightarrow 0$ while parameters $\alpha_{\text {eff }}^{\prime}, \tilde{v}, G_{o}, u, \tilde{x}^{\mu}$ with $(\mu=0,1,2,3)$ remain fixed. We would like to point out that insisting a decoupled gravity dual of NCOS allows only an infinitesimal boost $\gamma=\tilde{v} \epsilon$. This in turn says that a finite boost will not give a decoupled NCOS in the decoupling limit. Therefore, a Lorentz boost on a given NCOS will render it undecoupled. This is entirely consistent with the conclusion drawn in 14 in a different analysis. With the above, the gravity description is then

$$
\begin{aligned}
& d s^{2}=\epsilon h^{1 / 2}\left[\frac{u^{2}}{R^{2}}\left(-d \tilde{x}_{0}^{2}+d \tilde{x}_{1}^{2}+h^{-1}\left(d \tilde{x}_{3}^{2}+\left(d \tilde{x}^{2}-\tilde{v} d \tilde{x}^{0}\right)^{2}\right)\right)+\alpha_{\mathrm{eff}}^{\prime 2} R^{2}\left(\frac{d u^{2}}{u^{2}}+d \Omega_{5}^{2}\right)\right] \\
& e^{\phi}=G_{o}^{2} h^{1 / 2}, \quad F_{0123 u}=-\epsilon^{2} \frac{16 \pi n u^{3}}{\alpha_{\mathrm{eff}}^{\prime 2} R^{8} h} \\
& 2 \pi \alpha^{\prime} B=\epsilon \frac{u^{4}}{R^{4}} d \tilde{x}^{0} \wedge d \tilde{x}^{1} \\
& A_{2}=\epsilon \frac{u^{4}}{G_{o}^{2} R^{4} h}\left(d \tilde{x}^{2}-\tilde{v} d \tilde{x}^{0}\right) \wedge d \tilde{x}^{3}
\end{aligned}
$$

where

$$
h=1+\frac{u^{4}}{R^{4}}, \quad R^{4}=\frac{4 \pi n G_{o}^{2}}{\alpha_{\mathrm{eff}}^{\prime 2}} .
$$

It is not difficult to examine that the parameter $\tilde{v}$ looks like a velocity and this gravity description is related to that of NCOS with only space-time noncommutativity (corresponding to $\tilde{v}=0$ or resulting from the background without boost) by a Galilean transformation

$$
\tilde{x}^{0} \rightarrow \tilde{x}^{0}, \quad \tilde{x}^{2} \rightarrow \tilde{x}^{2}-\tilde{v} \tilde{x}^{0}
$$

Further, if we calculate the noncommutative parameters, we have

$$
\Theta^{01}=2 \pi \alpha_{\text {eff }}^{\prime}, \quad \Theta^{12}=-2 \pi \alpha_{\text {eff }}^{\prime} \tilde{v}
$$

which is consistent with the Galilean transformation given in Eq. ([1.6) above. Therefore, we in a gravity setup show that the V-duality conjecture proposed in 14 holds true. 


\section{THE GALILEAN NATURE OF NCYM: V-DUALITY}

The field theory analysis of NCYM for this case has been given in [14]. It concluded there that one cannot have NCYM with space-time noncommutativity in addition to the space-space one. This is also confirmed in other independent analysis, for example, in 15, 19. This clearly indicates that one cannot perform a Lorentz transformation on NCYM since it will in general lead to space-time noncommutativity. Any allowed transformation on the decoupled theory must be induced from the transformation of string theory before the decoupling. We will show in this section that the allowed one is again a Galilean transformation, describing the V-duality action. This is entirely consistent with the fact that we cannot have space-time noncommutativity for NCYM since a Galilean transformation on a space-space noncommutativity cannot give rise to a space-time one.

In this section, we will spell out the V-duality action for NCYM using its gravity dual description. The relevant gravity configuration is the (D1, D3) bound state [23, 25] boosted along a D3 brane direction perpendicular to the D-strings in the state? Its explicit form is

$$
\begin{aligned}
& d s^{2}=H^{-1 / 2}\left[-\left(d x^{0}\right)^{2}+\left(d x^{1}\right)^{2}+\left(d x^{2}\right)^{2}+\frac{H}{H^{\prime}}\left(d x^{3}\right)^{2}\right. \\
& \left.\quad+\frac{H-H^{\prime}}{H^{\prime}}\left(\cosh \gamma d x^{2}-\sinh \gamma d x^{0}\right)^{2}\right]+H^{1 / 2}\left[d r^{2}+r^{2} d \Omega_{5}^{2}\right], \\
& e^{\phi}=g_{s} \sqrt{H / H^{\prime}}, \quad F_{5}=16 \pi n \alpha^{\prime 2}\left(* \epsilon_{5}+\epsilon_{5}\right), \\
& 2 \pi \alpha^{\prime} B=H^{\prime-1} \tan \alpha\left[\sinh \gamma d x^{0} \wedge d x^{3}-\cosh \gamma d x^{2} \wedge d x^{3}\right] \\
& A_{2}=g_{s}^{-1} H^{-1} \sin \alpha\left[\cosh \gamma d x^{0} \wedge d x^{1}+\sinh \gamma d x^{1} \wedge d x^{2}\right]
\end{aligned}
$$

where $d s^{2}$ is the string metric and the harmonic functions $H$ and $H^{\prime}$ are also given by Eq. (II.2). We can read the D3 brane worldvolume electric and magnetic background fields from the asymptotic B-field as $E_{3}=B_{03}^{\infty}=\tan \alpha \sinh \gamma, B_{1}=B_{23}^{\infty}=-\tan \alpha \cosh \gamma$. Now we have $\mathbf{E}^{2}-\mathbf{B}^{2}=-\tan ^{2} \alpha \leq 0$ and $\mathbf{E} \cdot \mathbf{B}=0$ which are Lorentz invariant. Since we are interested in NCYM, $\tan \alpha$ is nonzero. So $\mathbf{E}^{2}-\mathbf{B}^{2}<0$, i.e., space-like as it should be for NCYM. The gravity dual of NCYM can be obtained from the above with the following decoupling limit:

\footnotetext{
${ }^{8}$ This boosted configuration was also given in 27 for the discussion of light-like NCYM.
} 


$$
\begin{aligned}
& \alpha^{\prime}=\alpha_{\text {eff }}^{\prime} \epsilon, \quad \cos \alpha=\epsilon, \quad \gamma=\tilde{v} \epsilon, \quad g_{s}=\epsilon G_{o}^{2}, \\
& r=\alpha_{\text {eff }}^{\prime} \epsilon u, \quad x^{0,1}=\tilde{x}^{0,1}, \quad x^{2,3}=\epsilon \tilde{x}^{2,3}
\end{aligned}
$$

where $\epsilon \rightarrow 0$ while parameters $\alpha_{\text {eff }}^{\prime}, \tilde{v}, G_{o}, \tilde{x}^{\mu}$ with $(\mu=0,1,2,3)$ remain fixed. With the above decoupling limit, the gravity dual of NCYM is

$$
\begin{aligned}
& d s^{2} / \epsilon=\frac{u^{2}}{R^{2}}\left[-d \tilde{x}_{0}^{2}+d \tilde{x}_{1}^{2}+h^{-1}\left(d \tilde{x}_{3}^{2}+\left(d \tilde{x}^{2}-\tilde{v} \tilde{d} \tilde{x}^{0}\right)^{2}\right)\right] \\
& +\alpha_{\mathrm{eff}}^{\prime 2} R^{2}\left(\frac{d u^{2}}{u^{2}}+d \Omega_{5}^{2}\right) \\
& e^{\phi}=G_{o}^{2} h^{-1 / 2}, \quad F_{0123 u}=-\epsilon^{2} \frac{16 \pi n u^{3}}{\alpha_{\mathrm{eff}}^{\prime 2} R^{8} h}, \\
& 2 \pi \alpha^{\prime} B=-\epsilon \frac{u^{4}}{R^{4} h}\left(d \tilde{x}^{2}-\tilde{v} d \tilde{x}^{0}\right) \wedge d \tilde{x}^{3}, \\
& A_{2}=\epsilon \frac{u^{4}}{G_{o}^{2} R^{4}} d \tilde{x}^{0} \wedge d \tilde{x}^{1}
\end{aligned}
$$

where $h$ and $R$ are defined the same as those in Eq. (ㅍ.5). We would like to point out again that the above decoupled gravity dual description of NCYM requires infinitesimal small boost parameter $\gamma=\tilde{v} \epsilon$. A finite boost would imply that we cannot have the decoupled theory. This further implies that a Lorentz boost acting on a given decoupled NCYM will render it undecoupled.

One can examine that the above gravity dual of NCYM is related to that of NCYM resulting from the decoupling in a purely magnetic background (corresponding to $\tilde{v}=0$ ) again by a Galilean transformation defined in Eq. ([I.6). If we calculate the noncommutativity parameters, we have, as expected,

$$
\Theta^{0 i}=0, \quad \Theta^{23}=2 \pi \alpha_{\mathrm{eff}}^{\prime},
$$

where $i=1,2,3$. This result is consistent with the Galilean transformation Eq. (II.6) as discussed at the outset of this section. So we show that V-duality also works for NCYM.

We are not surprised by the infinitesimal boost requirement for NCYM given that for NCOS discussed in the previous section. This is because the two are S-dual to each other which can be explicitly checked from the gravity description for NCYM given in this section and that for NCOS in the previous section. So the V-duality here is also a consequence of S-duality. However, the physical reason behind is not explained up to this point. We try to provide this before turning to the next section. 
As we know that to have a decoupled NCOS, we need to have a background electric field present from the world volume view. This electric field represents the presence of F-strings inside D3 branes. The stable BPS configuration is the so-called non-threshold bound state of (F, D3). On the other hand, for NCYM we need to have magnetic background which represents the presence of D-strings inside D3 branes such that they form stable BPS non-threshold (D1, D3) bound state. So for D3 branes, the presence of D-strings favors the decoupled NCYM while that of F-strings favors the decoupled NCOS. We know that the decoupling limit for one against that for other. One cannot form a consistent hybrid theory which contains both NCOS and NCYM while at the same time it decouples the bulk closed strings. The effect of a Lorentz boost, for example, acting on (F, D3) is to create Dstrings orthogonal to the F-strings in the bound state with its charge density proportional to the Lorentz boost. Given the previous discussion, we know that if the charge density for D-strings is comparable to that of F-strings in the bound state, we cannot have a decoupled NCOSF. Only for infinitesimally small D-string charge, a decoupled NCOS has a chance to exist. This is precisely what we achieved in the previous section and this is the physical reason why only a Galilean transformation or V-duality is allowed for the decoupled theory. The discussion for NCYM follows the same line but now the F-string charge density needs to be infinitesimal small, implying an infinitesimal boost.

\footnotetext{
${ }^{9}$ The best we can do is to infinitely boost a vanishing small electric background to end up with a new light-like NCYM [26,27]. The same applies for infinitely boosting a vanishing small magnetic background. As discussed in [26,27], the light-like NCYM, even though a noncommutative field theory, is qualitatively different from the usual NCYM with space-like noncommutativity, therefore a new kind of noncommutative field theory. One can say that NCOS, light-like NCYM and space-like NCYM result respectively from the decoupling of D3 branes in time-like, light-like and space-like electric and magnetic backgrounds. We stress that the aforementioned infinite boost is used just for defining a finite light-like background from a vanishing either time-like or space-like one so that a decoupling limit for light-like NCYM can be addressed. This infinite boost is not directly related to V-duality. We will address the related V-duality issue in [22].
} 


\section{HOLOGRAPHY AND NCOS}

A holographic correspondence between NCYM and its gravity dual was proposed in [28]. This was further extended in [14] to NCOS with pure time-space noncommutativity. It basically states that the radial profile of the on-shell closed string moduli (string metric, NSNS B-field and dilaton) in the dual gravity description of NCYM or NCOS can be derived using Seiberg-Witten relations [5] between fixed open string moduli (effective flat open string metric, noncommutative parameters and open string coupling) and the closed string moduli, provided a simple ansatz for the running string tension as the function of energy scale is assumed. Given V-duality, the holographic correspondence [28,14 is expected to hold for NCOS and NCYM discussed in this paper as well. In this section, we provide a direct demonstration of this correspondence for the case of NCOS as an independent and direct check for V-duality. The check for NCYM is even easier and we will not repeat it here.

Physically the holographic correspondence proposed in 28] for NCYM and in [14 for NCOS is a natural consequence of D-brane picture in the decoupling limit. We know that there are two physically equivalent descriptions of D-branes, one is that of the open string ending on the D-branes and the other is the closed string one. In general, from the closed string perspective, D-branes themselves interact with bulk closed string modes and from the open string perspective, the open string ending on the D-branes interacts with the same bulk modes. However, in the so-called decoupling limit, these two descriptions describe the dynamics of the same D-branes, therefore they must be physically equivalent. From the open string perspective, the worldvolume must be flat since the bulk gravity (or closed string) decouples. However, the closed string description of D-branes (i.e., the solitonic profile of D-branes in the decoupling limit) gives a curved background (loosely called the near-horizon geometry of D-brane volume obtained from the open string perspective that gives a holographic description of the above curved background.

\footnotetext{
${ }^{10}$ The original spacetime is separated to two regions: one is occupied by the bulk closed strings and the other describes the D-branes. The bulk closed strings cannot enter into the region of D-branes since its size is substringy. This is the picture of the decoupling of D-branes from the bulk closed strings in terms of the closed string (or bulk) description.
} 
A better description for a decoupled theory can be obtained when there are electric and/or magnetic background (or NSNS B-background) on the D-branes. In terms of this description, the D-brane metric seen by the end-points of the effective open strings does not coincide with the closed string metric. And this description gives rise to coordinate noncommutativity. In [5] Seiberg and Witten have established the general relations between closed string moduli and the effective open string ones. In general, these relations hold only to the leading order since a flat rigid geometry for D-branes is assumed there. However, in the decoupling limit for D-branes, these relations become exact since the bulk modes, which render the D-brane world volume curved, decouple. Originally Seiberg-Witten relations are suggested in the first description of the D-branes (perturbative open-strings ending on flat D-branes). The important recognition made in [28, 14] is that these relations apply also to the closed string moduli in the dual gravity description. This provides a better understanding of how the gravity dual describes noncommutative theories in a holographical way. We can understand this as follows.

The open string description is non-gravitational (since the bulk closed strings decouple). Therefore the worldvolume geometry should be independent of energy scale, so are the metric and the noncommutativity parameters $\square$. As we know, the energy scale in the open string description is just the radial coordinate $u$ in the closed string description of D-branes. So for a fixed $u$, we can ask for the effective description of open string. This effective open string coupling should be the same as before, since it is dimensionless, but the effective string tension should be determined properly. We therefore expect that Seiberg-Witten relations should hold between the closed string moduli at a fixed but arbitrary $u$ and the same open string moduli, but now for the effective open string with its yet unknown effective open string tension. The success of this prescription depends crucially on how to determine the effective string tension or $\alpha_{\text {run }}^{\prime}$. Given that the closed string moduli in original Seiberg-Witten relations are constants and independent of the $u$ coordinate, they must correspond to those outside of the boundary of the gravity description. This fact helps us to set the boundary condition for $\alpha_{\text {run }}^{\prime}$ which was also discussed in [28.14] in a different context. In other words, it must approach to its boundary value as $u \rightarrow \infty$.

\footnotetext{
${ }^{11}$ One may wonder if the noncommutative parameters can be so, since they are dimensionful. The correct derivations given in 28, 14] ensure this.
} 
In the following, we derive the NSNS fields for the gravity dual of NCOS given in Eq. ([1.4) as an example. The Seiberg-Witten relations [5] between open string moduli $\left(G_{\mu \nu}, \Theta^{\mu \nu}, G_{o}\right)$ and closed string ones $\left(g_{\mu \nu}, B_{\mu \nu}, g_{s}\right)$ in their original forms are

$$
\begin{gathered}
G_{\mu \nu}=g_{\mu \nu}-\left(2 \pi \alpha^{\prime}\right)^{2}\left(B g^{-1} B\right)_{\mu \nu}, \\
\Theta^{\mu \nu}=2 \pi \alpha^{\prime}\left(\frac{1}{g+2 \pi \alpha^{\prime} B}\right)_{A}^{\mu \nu},
\end{gathered}
$$

and

$$
G_{s}=G_{o}^{2}=g_{s}\left(\frac{\operatorname{det} G}{\operatorname{det}\left(g+2 \pi \alpha^{\prime} B\right)}\right)^{1 / 2} .
$$

In the above, ()$_{A}$ denotes the anti-symmetric part and $\mu=0,1,2,3$.

The open string metric $G_{\mu \nu}$ and the noncommutative parameters $\Theta^{\mu \nu}$ can be calculated using the asymptotic values for closed string metric and NSNS B-field from Eq. ([I.1) with respect to the scaled coordinates $\tilde{x}^{\mu}$ as

$$
G_{\mu \nu}=\epsilon\left(\begin{array}{cccc}
-\left(1-\tilde{v}^{2}\right) & 0 & -\tilde{v} & 0 \\
0 & 1 & 0 & 0 \\
-\tilde{v} & 0 & 1 & 0 \\
0 & 0 & 0 & 1
\end{array}\right),
$$

and

$$
\Theta^{01}=2 \pi \alpha_{\text {eff }}^{\prime}, \quad \Theta^{12}=-2 \pi \alpha_{\text {eff }}^{\prime} \tilde{v} .
$$

We know that the open string metric $G_{\mu \nu}$ is defined with respect to the string tension $1 /\left(2 \pi \alpha^{\prime}\right)$. We can redefine a new open string metric $G_{\mu \nu}^{\prime}=G_{\mu \nu} / \epsilon$ with a finite tension $1 /\left(2 \pi \alpha_{\mathrm{eff}}^{\prime}\right)$ since $\epsilon=\alpha^{\prime} / \alpha_{\mathrm{eff}}^{\prime}$. We now have $\alpha^{\prime} G^{\mu \nu}=\alpha_{\mathrm{eff}}^{\prime} G^{\mu \nu}=$ fixed as expected. The new metric $G_{\mu \nu}^{\prime}$ can be obtained from Eq. (IV.1) simply by setting closed string metric $g \rightarrow g / \epsilon$ and $\alpha^{\prime} \rightarrow \alpha_{\text {eff }}^{\prime}$. In other words, we now work with the metric $d s^{2} / \epsilon$ and string constant $\alpha_{\mathrm{eff}}^{\prime}$. So $\alpha_{\mathrm{eff}}^{\prime}$ is the boundary value for the $\alpha_{\text {run }}^{\prime}$. The question is how to obtain a correct ansatz for $\alpha_{\text {run }}^{\prime}$.

Consider a type IIB F-string probe in a given background, its kinetic term is

$$
\frac{1}{2 \pi \alpha^{\prime}} \int d^{2} \sigma \partial_{\alpha} x^{M} \partial^{\alpha} x^{N} g_{M N},
$$


with $g_{M N}$ the bulk metric. Now consider this string to probe the D3 brane background. Recall that the NCOS tension is the same with respect to its metric in any direction. But the story is different if we try to read the running tension from the closed string side. The reason that we have a decoupled NCOS is because the near-critical electric force balances the original string tension to end up with a finite tension if the original tension is sent to infinity. This NCOS is now along $x^{1}$ direction. It is in this direction that the open string metric is different from the asymptotic closed string one. One cannot use the probe F-string to read the running tension for the effective open string along this direction. Examining the open string metric $G_{\mu \nu}$ given in Eq. (IV.4), we can see that the open string metric $G_{22}, G_{33}$ are the same as the corresponding asymptotic closed metric $g_{22}, g_{33}$ with respect to the scaled coordinates $\tilde{x}^{\mu}$. We should be able to read the running tension for the effective open string using the probe along, for example, $\tilde{x}^{3}$ direction. In other words, from $1 /\left(2 \pi \alpha^{\prime}\right) \int \partial_{\alpha} \tilde{x}^{3} \partial^{\alpha} \tilde{x}^{3} g_{33}(u)=g_{33}(u) /\left(2 \pi \alpha^{\prime}\right) \int \partial_{\alpha} \tilde{x}^{3} \partial^{\alpha} \tilde{x}^{3}$, we have $\alpha_{\text {run }}^{\prime}=\alpha^{\prime} / g_{33}$. From Eq. ([II.4), we have $\alpha_{\text {run }}^{\prime}=\alpha_{\text {eff }}^{\prime} R^{2} h^{1 / 2} / u^{2}$. It is easy to see that $\alpha_{\text {run }}^{\prime} \rightarrow \alpha_{\text {eff }}^{\prime}$ as $u \rightarrow \infty$, i.e., satisfying the boundary condition as expected. The physical picture above is: The closed string has a string constant $\alpha^{\prime}$ but moving in a curved background, for example, $g_{33}$ (or $\alpha_{\text {eff }}^{\prime}$ but in $g_{33} / \epsilon$ ) while the open string has a flat metric $G_{\mu \nu}$ but with a running $\epsilon \alpha_{\text {run }}^{\prime}$ (or $G_{\mu \nu}^{\prime}$ with $\left.\alpha_{\text {run }}^{\prime}\right)$.

With the above understanding and given

$$
\alpha_{\text {run }}^{\prime}=\alpha_{\text {eff }}^{\prime} R^{2} h^{1 / 2} / u^{2},
$$

we now present the holographic derivation for the NSNS fields in the gravity dual of NCOS given in Eq. (II.4). Given that the gravity dual of NCOS in (III.4) is obtained from a background with orthogonal electric and magnetic fields and the magnetic background is brought about by a Lorentz boost, we expect that the only nonvanishing metric along the brane directions are $g_{00}, g_{11}, g_{02}=g_{20}, g_{22}=g_{33}$. We also have possible nonvanishing $B_{01}$ and $B_{12}$. In the following, we use open string metric $G_{\mu \nu}^{\prime}=G_{\mu \nu} / \epsilon$, this implies that we use closed string metric $g / \epsilon$. Given the ansatz for $\alpha_{\text {run }}^{\prime}$, it implies that we know $g_{22} / \epsilon=g_{33} / \epsilon=\alpha_{\text {eff }}^{\prime} / \alpha_{\text {run }}^{\prime}$ as is evident from the above discussion. Now the $\alpha^{\prime}$ in Eqs. (IV.1) - (IV.3) is replaced by $\alpha_{\mathrm{run}}^{\prime}$. This further implies that the closed string metric used in Seiberg-Witten relations should be $\bar{g}_{\mu \nu}=g_{\mu \nu} / g_{33}$. With the above in mind, we set $\bar{g}_{00}=-f_{1}, \bar{g}_{11}=f_{2}, \bar{g}_{02}=f_{3}$ and $\bar{g}_{22}=\bar{g}_{33}=1$. For simplicity, we set $2 \pi \alpha_{\text {run }}^{\prime} B_{01}=$ $h_{1}, 2 \pi \alpha_{\text {run }}^{\prime} B_{12}=h_{2}$. Then Seiberg-Witten relations give, from the metric, 


$$
1+\frac{h_{2}^{2}}{f_{2}}=1, \quad f_{3}=-\tilde{v}, \quad f_{1}-\frac{h_{1}^{2}}{f_{2}}=1-\tilde{v}^{2}, \quad f_{2}-\frac{h_{1}^{2}}{f_{1}+f_{3}^{2}}=1,
$$

and, from the noncommutative matrix,

$$
\frac{h_{1}}{h_{1}^{2}-f_{2}\left(f_{1}+f_{3}^{2}\right)}=-\frac{u^{2}}{R^{2} h^{1 / 2}} .
$$

One can solve from the above to have

$$
f_{1}=h-\tilde{v}^{2}, \quad f_{2}=h, \quad f_{3}=-\tilde{v}, \quad h_{1}=\frac{u^{2}}{R^{2}} h^{1 / 2}, \quad h_{2}=0 .
$$

One can check that these solutions give the correct metric and NSNS B-field in Eq. (ㅍ.4). For example, we have $2 \pi \alpha_{\text {run }}^{\prime} B_{01}=h_{1}$ which gives $2 \pi \alpha_{\text {eff }}^{\prime} B_{01}=u^{4} / R^{4}$, the correct answer. Using the above results, we can calculate the effective closed string coupling $e^{\phi}$, from $e^{\phi}=G_{o}^{2}\left[-\operatorname{det}\left(\bar{g}+2 \pi \alpha_{\text {run }}^{\prime} B\right)\right]^{1 / 2}$, as

$$
e^{\phi}=G_{o}^{2} h^{1 / 2}
$$

again the correct answer. We note that the running string tension for NCOS in our case, Eq. (IV.7), as a function of the radial coordinate $u$ is the same as that given in ref. [14 for NCOS with pure space-time noncommutativity. This means that the NCOS's related by V-duality have the same running string tension. Or the latter remains unchanged under V-duality. This may be viewed as an additional evidence for V-duality.

\section{CONCLUSIONS AND DISCUSSIONS}

Before concluding this paper, let us now address an issue regarding the supporting argument for the equivalence of the noncommutative theories that arise from decoupling D-branes in Lorentz-boost related backgrounds. This issue arises as follows: In the usual perturbative open string description of D-branes, from the outset, one assumes that Dbrane are rigid and flat. This is known to be not exactly true, since the coupling to bulk closed strings will render the D-branes curved. In this sense, the Poincare symmetry along the brane directions in this description should be considered true only approximately. However, in a limit in which the bulk closed strings decouple, this symmetry is expected to become exact. Moreover, the decoupling for a noncommutative theory (either NCOS or NCYM for the purpose of this paper) in general requires a D-brane worldvolume electric and/or magnetic background field. The presence of such a background field breaks the 
worldvolume Lorentz symmetry, but this breakdown is only spontaneous. Namely, if we simultaneously Lorentz transform all backgrounds, including the electromagnetic one, we end up with a physically equivalent situation. This can be explicitly seen in our present study: in the gravity dual description in Sec. 2 and 3 it is obvious that the Lorentz boost on a relevant D-brane bound state solution before decoupling leads to a physically equivalent solution. Thus, given that the worldvolume Lorentz symmetry for the parent open string theory should be exact in decoupling limits and that the introduction of backgrounds breaks the symmetry only spontaneously, we expect that the noncommutative theories of Dp branes resulting from decoupling in Lorentz-boost related background fields are physically equivalent.

In conclusion, we test the V-duality conjecture proposed in [14] for NCOS using its gravity dual description. We also extend the V-duality for NCYM and find that this seems to be true in general. The implication of this V-duality is that one can no longer use a Lorentz transformation other than a Galilean one, for example, to choose a particular frame in performing calculations in NCOS and NCYM. We present an explanation for the holographic correspondence given in [28, 114] for noncommutative theories, and give a holographic derivation for the gravity dual of NCOS discussed in this paper. In addition, our holographic derivation shows that the NCOS's (or NCYM's) related by V-duality share the same running string tension as a function of the radial coordinate (or energy scale).

The Galilean nature of this V-duality is, in a sense, implied already by the noncommutative relation $\left[x^{\mu}, x^{\nu}\right]=i \Theta^{\mu \nu}$, which implies spacetime nonlocality. This in turn implies that we have action at distance. In other words, we do not have a limit on the speed of light巴, i.e., we are dealing with Newtonian-like physics. With the V-duality discussed in this paper and the above simple physical implication behind the noncommutative relations, one can easily understand the recent finding in 29, 30 that solitons can travel faster than the speed of light in NCYM and also the infinitely large speed of light as discussed in [31 33]. We will elaborate this and other related points further in the forthcoming paper [22].

\footnotetext{
${ }^{12}$ There are some subtleties involved when the boost is not orthogonal to the space-time noncommutative directions. We will address this in detail in [22].
} 


\section{ACKNOWLEDGEMENTS}

The work of RGC was supported in part by the Japan Society for Promotion of Science and in part by Grants-in-Aid for Scientific Research Nos. 99020 and 12640270, and by a Grant-in-Aid on the Priority Area: Supersymmetry and Unified Theory of Elementary Particles. JXL acknowledges the support of U. S. Department of Energy. The research of YSW was supported in part by the U.S. National Science Foundation under Grant No. PHY-9907701. 


\section{REFERENCES}

[1] P. M. Ho and Y. S. Wu, "Noncommutative Geometry and D-branes", Phys. Lett. B398, 52 (1997), hep-th/9611233.

[2] A. Connes, M. Douglas and A. Schwartz, "Noncommutative geometry and matrix theory", JHEP 9802, 003 (1998), hep-th/9711162.

[3] M. R. Douglas and C. Hull, "D-branes and the noncommutative Torus", JHEP 9802, 008 (1998), hep-th/9711165.

[4] P. M. Ho and Y.S. Wu, "Noncommutative gauge theories in Matrix theory", Phys. Rev. D58, 026006 (1998), hep-th/9712201.

[5] N. Seiberg and E. Witten, "String theory and noncommutative geometry", JHEP 9909, 032 (1999), hep-th/9908142.

[6] A. Hashimoto and N. Itzhaki, "Non-commutative Yang-Mills and the AdS/CFT correspondence",Phys. Lett. B465, 142 (1999), hep-th/9907166.

[7] J. Maldacena and J. Russo, "Large N limit of noncommutative gauge theories", JHEP 9909, 025 (1999), hep-th/9908134.

[8] M. Alishahiha, Y. Oz and M. Sheikh-Jabbari, "Supergravity and large N noncommutative field theories", JHEP 9911, 007 (1999), hep-th/9909215.

[9] N. Seiberg, L. Susskind and N. Toumbas, "Strings in background electric field space/time noncommutativity and a new noncritical string theory", JHEP 0006, 021 (2000), hep-th/0005040.

[10] R. Gopakumar, J. Maldacena, S. Minwalla and A. Strominger, "S-duality and noncommutative gauge theory", JHEP 0006, 036 (2000), hep-th/0005048.

[11] J. Barbon and E. Rabinovici, "Stringy Fuzziness as the Custodian of Time-Space Noncommutativity", Phys. Lett. B486, 202 (2000), hep-th/0005073.

[12] T. Harmark, "Supergravity and space-time noncommutative open string theory", JHEP 0007, 043 (2000), hep-th/0006023.

[13] I. Klebanov and J. Maldacena, " $1+1$ dimensional NCOS and its $U(N)$ gauge theory dual", hep-th/0006085. 
[14] G. -H. Chen and Y. -S. Wu, "Comments on noncommutative open string theory: V-duality and holography", hep-th/0006013.

[15] J.X. Lu, S. Roy and H. Singh, "((F,D1), D3) bound state, S-duality and non-commutative open string/Yang-Mills theory", JHEP 0009, 020 (2000), hepth/0006193.

[16] J. Russo and M. Sheikh-Jabbari, "On noncommutative open string theories", JHEP 0007, 052 (2000), hep-th/0006202.

[17] S.-J. Rey and R. von Unge, " S-duality, noncritical open string and noncommutative gauge theory", hep-th/0007089.

[18] R.-G. Cai and N. Ohta, "(F1,D1, D3) Bound State, Its Scaling Limits and $S L(2, Z)$ Duality", Prog. Theor. Phys. 104, 1073 (2000), hep-th/0007106.

[19] J. X. Lu, S. Roy and H. Singh, "SL (2, Z) duality and 4-dimensional noncommutative theories", hep-th/0007168 (to appear in NPB).

[20] N. Seiberg, L. Susskind and N. Toumbas, "Space/Time noncommutativity and causality", JHEP 0006, 044 (2000), hep-th/0005015.

[21] J. Gomis and T. Mehen, "Space-time noncommutative field theories and unitarity", Nucl. Phys. B591, 265 (2000), hep-th/0005129.

[22] R.-G. Cai, J. X. Lu and Y.-S. Wu, in preparation.

[23] J. G. Russo and A. A. Tseytlin, "Waves, boosted branes and BPS states in M-theory", Nucl. Phys. B490 (1997) 121.

[24] J. X. Lu and S. Roy, "Non-threshold (F, Dp) bound states", Nucl. Phys. B560 (1999) 181, hep-th/9904129.

[25] J. Breckenridge, G. Michaud and R. Myers, "More D-brane bound states", Phys. Rev. D55 (1997) 6438.

[26] O. Aharony, J. Gomis and T. Mehen, "On theories with light-light noncommutativity", JHEP 0009, 023 (2000), hep-th/0006236.

[27] M. Alishahiha, Y. Oz and J. Russo, "Supergravity and Light-like Noncommutativity", JHEP 0009, 002 (2000), hep-th/0007215. 
[28] M. Li and Y.-S. Wu, "Holography and Noncommutative Yang-Mills", Phys. Rev. Lett. 84, 2084 (2000), hep-th/9909085.

[29] A. Hashimoto and N. Itzhaki, "Traveling Faster than the speed of light in Noncommutative Geometry", hep-th/0012093.

[30] D. Bak, K. Lee and J.-H. Park, "Noncommutative Vortex Solitons", hep-th/0011099.

[31] U. Danielsson, A. Goijosa and M. Kruczenski, " Newtonian Gravitons and D-brane Collective Coordinates in Wound String Theory", hep-th/0012183.

[32] U. Danielsson, A. Goijosa and M. Kruczenski, "IIA/B Wound and Wrapped", JHEP 0010, 020 (2000), hep-th/0009182.

[33] J. Gomis and H. Ooguri, "Non-relativistic Closed String Theory", hep-th/0009181. 